Technical Communication

\title{
Development of a Path Generation Algorithm for Large-Area Laser Patterning Using a Manual-Input Control-Point
}

\author{
Kwangho Yoon ${ }^{1}$, Kyunghan $\mathrm{Kim}^{1}$ and Jaehoon Lee ${ }^{* 1}$ \\ ${ }^{* 1}$ Dept. of High Density Energy Beam Processing \& System, KIMM, 156 Gajeonbuk-ro, Yuseong-gu, \\ Daejeon, Republic of Korea 305-343 \\ E-mail: jaholee@kimm.re.kr
}

In this study, we have developed stage path generation algorithm to extend scanner fabrication area into the stage working area. Proposed a manual input control point (MICP) path algorithm is to teach the stage path by a control point manually. G-code is a programming language for CNC machines. The conventional system is difficult to use because it requires G-code inputs and takes a long time to prepare for processing when the data volume is high. But proposed the stage-path algorithm with a non-uniform rational B-spline (NURBS) curve uses the mouse pointer to receive control-point (CP) inputs for path generation. It also reduces the processing time because helps the user to make the desired path. The speed profile is automatically calculated using the constraints, and a smooth profile is determined by defining the maximum acceleration/deceleration. A device for storing $10 \mathrm{us} / 32$-bit data was developed to verify that $350 \times 350 \mathrm{~mm}$ squares could be processed without exceeding the $50 \times 50 \mathrm{~mm}$ scan area. In addition, the two created path types were used to measure processing precision, and errors were comparatively analyzed according to path type.

DOI: $10.2961 / \mathrm{jlmn} .2015 .02 .0023$

Keywords: Laser, Large-Area, Scanner, Path Generation.

\section{Introduction}

Recently, the demand for large-screen displays has been rapidly increasing. Such products require continuous largearea microprocessing for light-guide plates or optical film. In the microprocessing field, laser processing with ultrashort wave pulses is used as an eco-friendly and noncontact process [1-3]. Although laser processing with a scanner is very fast, its processing area is limited. Laser processing with a stage can be used over a large area, but it is difficult to uniform laser pulse irradiation for high-speed processing because the stage is generally heavy system and it has higher acceleration/deceleration capability. So the step-and-scanning method was developed by combining advantages of two individual systems for large-area laser processing. In this method, a scanner and a stage are used. The scanner is used to process a limited area, after which the stage moves. Repetition of this process makes largearea laser processing possible; however, it creates discontinuous points. To overcome this disadvantage, the on-thefly method synchronizes the scanner and the stage. This enables continuous large-area laser processing and uniform processing [4-11].

Manufacturers such as ESI, LPKF, and AEROTECH sell products that use the on-the-fly method. These products have their own software and reduce the processing time by approximately $20 \%$. However, they require G-code inputs, so only experts have access to them. Moreover, if the amount of data increases, the longer is the preparation time for processing [12-14].

In this paper, a system and an algorithm are proposed for continuous large-area laser processing. A hybrid-type scanner and stage for high-speed processing are presented with an algorithm developed to determine the processing path and speed using the automatic alignment function and a mouse instead of the inconvenient G-code inputs. In the program of the machining center, G-code cannot be used if there is no major and easy access. Also, it has the disadvantage that the preparation time required for data processing is increased. The NURBS technique is used to draw the stage path, and the control points (CPs) are input using the mouse to obtain the user-desired path. In addition, constraint points are set to automatically calculate the speed for the stage processing paths. A $350 \times 350 \mathrm{~mm}$ square was processed in experiments conducted for multiple paths to verify the performance of the MICP path algorithm. And the processing precision was measured with many path types.

\section{On-the-Fly Concept and Configuration}

\subsection{On-the-Fly Concept}

For continuous large-area laser processing, the scanner must be linked to the stage, and the processing speed must be constant.

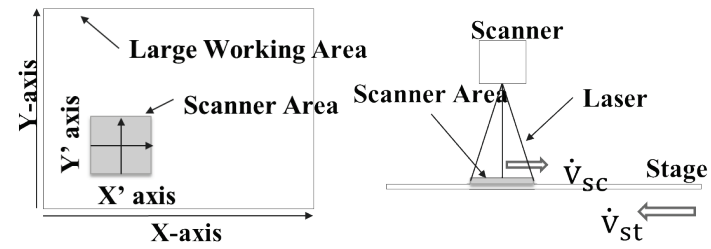

(a) Top view

(b) Side view

Fig. 1 On-the-fly concept. 
Figure 1 shows the on-the-fly concept for large-area processing, and Fig. 1 (a) shows the top view of the processing area. To link the scanner with the stage, the $\mathrm{X}$ - axis of the scanner must be aligned with the $\mathrm{X}$-axis of the stage, and the Y- axis of the scanner must be aligned with the Y axis of the stage. Fig. 1 (b) shows the speed relationship in the side view. To keep the processing speed constant, the vector sum of the processing speed of the scanner $\left(\dot{v}_{s c}\right)$ and the stage travel speed $\left(\dot{v}_{s t}\right)$, which is the total processing speed $\left(\dot{v}_{t}\right)$, must be constant:

$$
\dot{v_{t}}=\dot{v}_{s c}+\dot{v}_{s t},
$$

where $\dot{v}_{t}$ is constant

\subsection{On-the-Fly Configuration and Synchronization}

Continuous large-area laser processing requires three parts, as shown in Fig. 2. First, a high-speed processing scanner system with two galvanometers allows high-speed processing within a limited area via laser reflection. Second, the stage system of a hybrid-type enables large-area processing. Third, control boards are used to control the scanner and stage systems; the scan board delivers the marking signals to the scanner head. The marking on-thefly(MOTF) is a board for connecting the scanner with the stage and transmits the position information of the stage to the scanner.

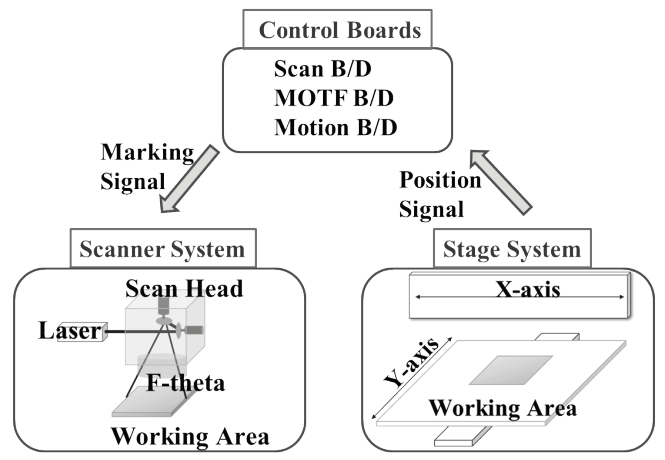

Fig. 2 System configuration.

Figure 3 shows a block diagram of the continuous large-area laser processing. The CAD data are inputted to the scan board and the MICP path algorithm. The scan board transmits the positional information to the scan header to move the galvanometer mirror.

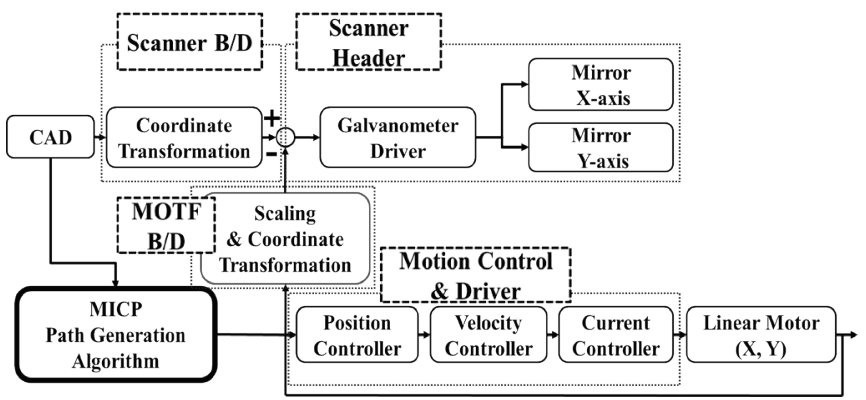

Fig. 3 Block diagram of the on-the-fly system.
The position and speed of the stage are determined by the MICP path algorithm, and the signal is transmitted to the motion board. To synchronize the scanner with the stage, the signal of the stage is transmitted to the scan board through the scale and coordinate system conversion, which is conducted in the MOTF $\mathrm{B} / \mathrm{D}$, and the results are compared with the input $\mathrm{CAD}$ to move the galvanometer mirror on the scanner header.

To utilize the scanner and stage, and for the processing of a large area, the coordinates of the stage and scanner must match. The processing of the desired size is made possible by the use of the ratio of the distance per pulse of the scanner and stage. The command signals of the scanner and stage are transmitted simultaneously from the origin of the specimen on the stage using a trigger signal.

Figure 4 shows the system for measuring the synchronized signals of the scanner and the stage. The signals transmitted to the scanner header are branched, and the signals of the scanner and stage are stored. The 32-bit data are stored at 10 us intervals, and communication is conducted based on the LAN. The board has two channels for storing data from the scanner and the stage separately. When the movements of the scanner and the stage are synchronized by this storage device, the scanner moves within the scan area, and the profiles, such as the speed and acceleration, can be identified.

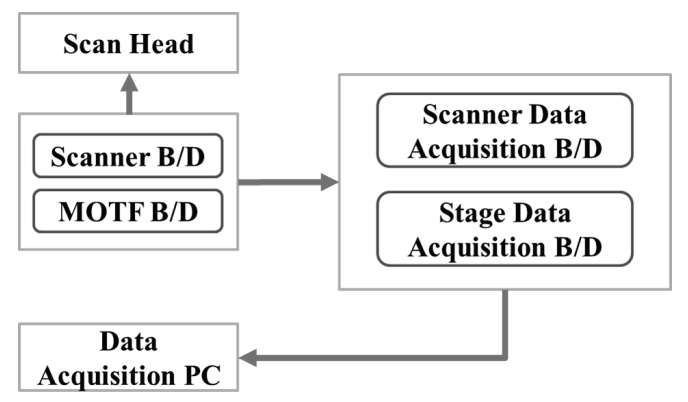

Fig. 4 Block diagram of scanner and stage data acquisition.

\section{Path Generation Using the MICP}

Figure 5 shows the sequence of the MICP path algorithm. When the CAD data are input, the nesting process is conducted (1). The nesting arranges the processing drawing, after which the starting points of the CAD drawing and the processing sequence, when there are multiple members, are determined (2). The stage path is created to continuously process the large area (3)). It is important to ensure that the stage path is smooth, considering the acceleration and deceleration. Accordingly, spline curves are often used. The spline curves are divided into interpolating and approximating curves according to whether or not the curves pass through the control points. In this study, NURBS curves were used as the spline curves to ensure smooth passage.

The NURBS curve is expressed using parameters. The B-spline, which is the basis of the NURBS, is defined as the control point, which is not passed through, and the end 
point, unlike the simple spline. The B-spline is expressed using the blending function $N_{i, k}$ shown in Equation 2:

$$
\mathrm{P}(\mathrm{u})=\sum_{\mathrm{i}=0}^{\mathrm{n}} N_{i, k}(u) P_{i},
$$

where $P$ is the control point, $u$ is a parameter knot vector, $n$ is a (control point-1), $k$ is the order, $u_{i}$ is the knot for the configuration of the blending function, and $N_{i, k}$ is a blending function of the $(k-1)$ order. The $i$-th blending function $N_{i, k}(u)$ is recursively calculated as

$$
\begin{gathered}
N_{i, 1}(u)=\left[\begin{array}{cc}
1 & u_{i} \leq u \leq u_{i+1} \\
0 & \text { otherwise }
\end{array}\right], \\
N_{i, k}(u)=\frac{\left(u-u_{i}\right)}{\left(u_{i+k-1}-u_{i}\right)} N_{i, k-1}(u)+\frac{\left(u_{i+k}-u\right)}{\left(u_{i+k}-u_{i+1}\right)} N_{i+1, k-1}(u)
\end{gathered}
$$

The B-spline curve has a convex hull and a normalizing property. If a B-spline is normalized, the blending function meets the following equation:

$$
\sum_{i=0}^{n} N_{i, k}(u)=1
$$

The knot $U=\left[u_{0}, \ldots, u_{m}\right]$ must meet the following conditions:

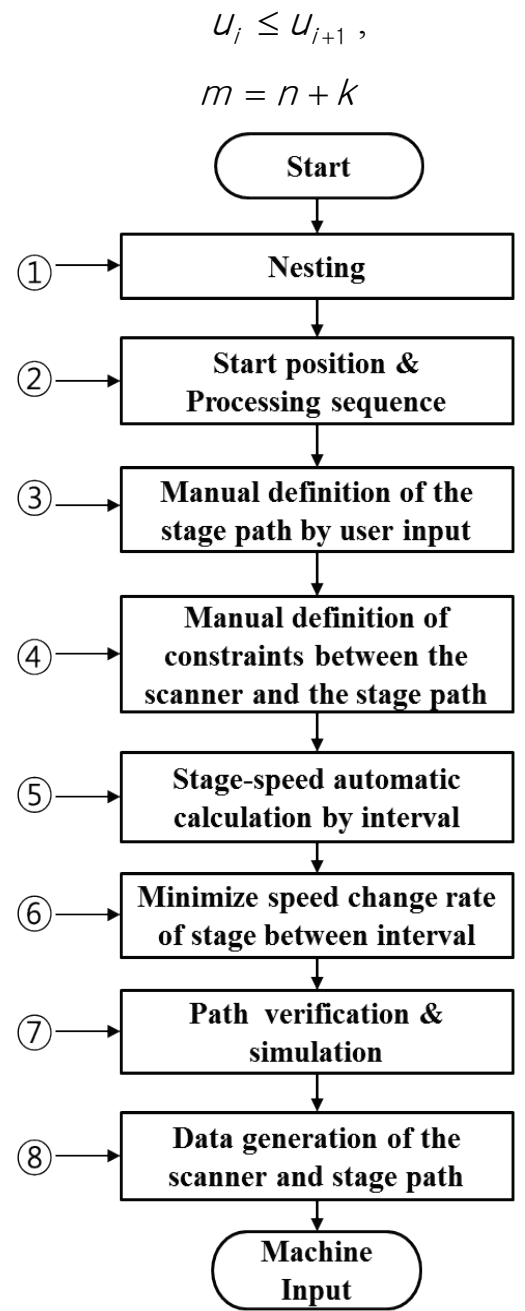

Fig. 5 Flowchart of path generation.
B-spline curves are classified into several types according to whether or not the numbers and configurations of the knots are uniform or periodical. NURBS is so called because the intervals between the knot values that compose the B-spline are non-uniform. The non-uniform knot values are mostly used in two types of situations: first, when the start and end points must be passed through to create a shape, and second, when sharp or bent edges are required between the control points, contrary to the smoothness of the B-spline curve. The NURBS curve is suitable for laser cutting because the start and end points must meet. The mathematical continuity, which is represented by the smoothness of the curve, is determined by the connection condition when two curve segments meet. The connection point of the B-spline can be easily identified from the configuration of the knot, and a curve with an order has a continuity at the connection point. At this time, one duplicate knot value reduces the continuity by one order. Therefore, the knot value can be duplicated as needed to produce a sharp or bent point in the middle of a curve. The following equation represents a rational B-spline curve with an inserted weighted value. The condition $W_{i} \geq 0$ is true at all times:

$$
P(u)=\frac{\sum_{i=0}^{n} N_{i, k}(u) P_{i} W_{i}}{\sum_{i=0}^{n} N_{i, k}(u) W_{i}}
$$

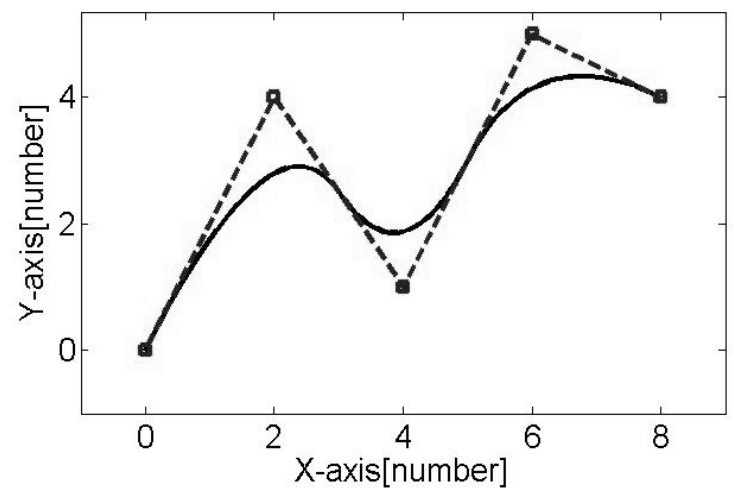

(a)

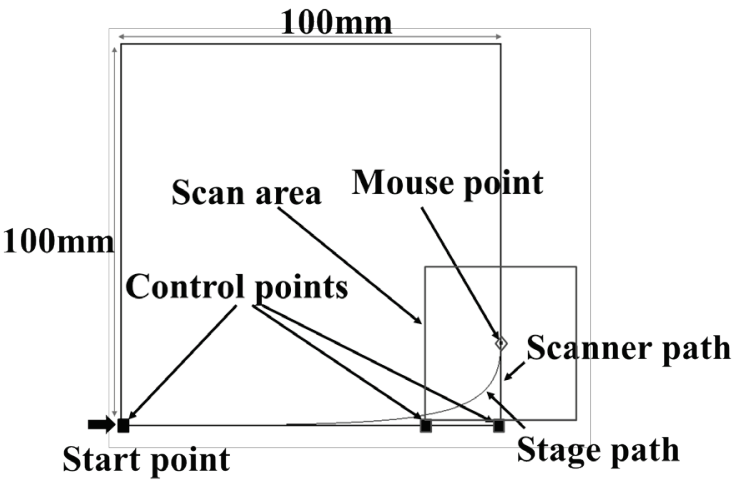

(b)

Fig. 6 Path generation using MICP. (a) NURBS curve example using MATLAB. (b) Actual path generation method. 
In a homogeneous coordinate system, the control point can be represented as a form of $\left(x_{i} \cdot W, y_{i} \cdot W, z_{i} \cdot W, W\right)$, and the degree of freedom can be increased with the concept of a weighted value for the curve design. NURBS is very useful for expressing free curves. However, the NURBS equation (2) requires numerous repeated operations when it has more control points and a higher curve degree. There is no problem with free offline 3D graphics, such as the CAD system, but it is advantageous for the interpolator to reduce and simplify long loops and operations, such as multiplication, division, and mathematical functions. In this study, a third-degree NURBS curve was used.

Figure 6 (a) shows the NURBS curve obtained by using MATLAB when $P_{i}=[0,2,4,6,8 ; 0,4,1,5,4], u_{i}=[0,0$, $0,1,2,3,3,3]$, and $W_{i}=[1,1,1,1,1]$.

The NURBS curve passes through the start and end points, but not through the control points, to create a smooth stage path; therefore, it is suitable to create a stage path. Figure 6 (b) shows the method of actually creating a stage path based on this method. In the MICP path algorithm, the control points are received from the mouse inputs, and the stage path is continuously created.

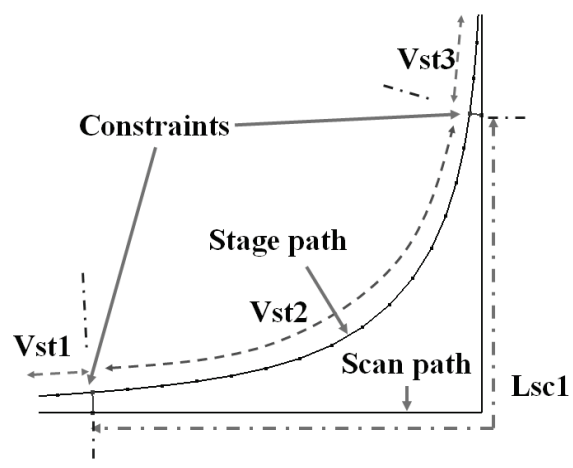

Fig. 7 Stage speed generation using MICP.

The stage path must be smooth near the edge to reduce the acceleration/deceleration. On the contrary, the scanner can move at a high speed near the edge. Because the scanner and the stage are synchronized, the scanner can process other parts using the position data of the stage. Figure 7 shows the instructions to generate the speed of the stage.

The stage speed is determined (5). Because the stage is synchronized with the scanner, the stage speed must be configured so that the laser does not go beyond the processing area of the scanner. Accordingly, the constraints are introduced to the actual processing path and the stage path after the generation of the stage path. As a constraint, one point on the stage path is connected to one point on the scanner path using the mouse. The equation for the stage speed is written as

$$
v_{t}=\frac{L_{s c 1}}{t} .
$$

If the total processing speed is given, the time can be calculated because the length of $L_{s c 1}$ is known. That is, the stage must move within $v_{s t 2}$ in time $t$. Because the length and time of the stage movement are given, the speed for section $v_{s t 2}$ is automatically calculated.

The stage acceleration is smoothly recreated (6)). For example, if there are large differences in the stage speeds, $v_{s t 1}, v_{s t 2}$, and $v_{s t 3}$, the stage acceleration will temporarily change significantly. To avoid this phenomenon, the stage path is divided at uniform intervals to allow step-by-step, smooth changes in the stage speed. The user inputs the maximum speed variation of the stage $\left(v_{\max }\right)$ and the point interval of the stage path to ensure slow changes. The following equation represents the coefficient of the changing speeds. A smaller maximum speed variation results in a smoother speed change:

$$
<N>=\frac{v_{s t 2}-v_{s t 1}}{V_{\max }} .
$$

A simulation is carried out to determine whether the stage path goes beyond the scanner processing area in the entire process (7)). Finally, the scanner path and the stage path are created (8). The data are transmitted to the scan board and the motion board for processing.

\section{Experiments}

\subsection{Experiment equipment and method}

The experiment equipment had a 2-axis stage and 2axis scanner galvanometers. The laser oscillator had a $5 \mathrm{~W}$ power, 355 wavelength at $30 \mathrm{~nm}, 20-100 \mathrm{kHz}$ repetition rate, and $1 \mathrm{~mm}$ beam size. Scanlab's IntelliScan 10 was used as the scanner, and a linear motor that had a $1.2 \mathrm{~m} / \mathrm{s}$ maximum speed, $15 \mathrm{~m} / \mathrm{s}^{2}$ maximum acceleration, $\pm 3 \mu \mathrm{m}$ accuracy, $\pm 1 \mu \mathrm{m}$ repeatability, $10 \mu \mathrm{m}$ straightness, and 10 $\mu \mathrm{m}$ flatness was used for the stage. The scanner area was $50 \times 50 \mathrm{~mm}$. To measure the synchronized signals of the scanner and the stage, 2 channels each for the scanner and the stage were used to store 32-bit data at 10 us. The product of the laser processing was measured using Mitutoyo's QuickVision Stream with $0.1 \mu \mathrm{m}$ precision. Figure 8 presents the experimental setup.

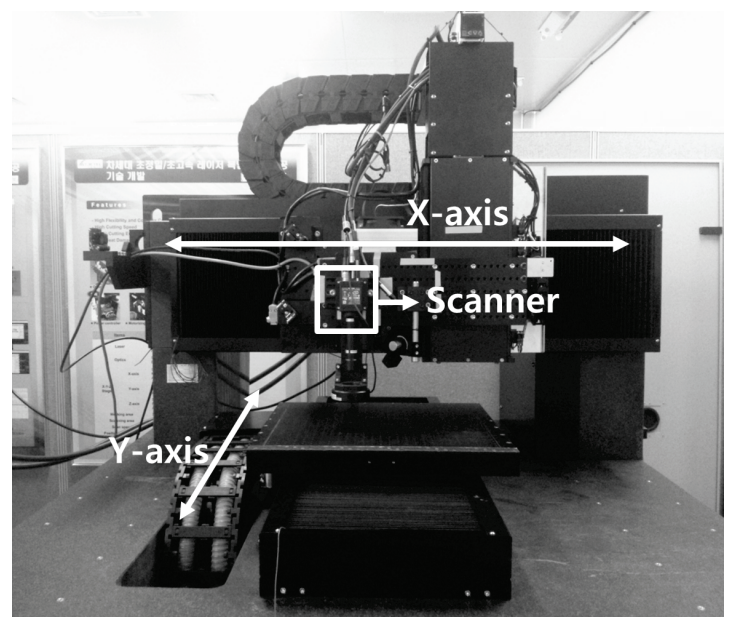

Fig. 8 Experimental setup. 
In this study, a $350 \times 350 \mathrm{~mm}$ square was tested. The data obtained from the proposed MICP path algorithm were applied to the actual system to analyze the signals. The speed was set at $400 \mathrm{~mm} / \mathrm{s}$ when the scanner and stage signals were measured. Using many paths that could be created in the CAD drawing with the proposed paths, the precision values of the actual product in the two cases in Fig. 9 were measured. Figure 9 (a) shows the stage path that was centered in the scanner area, and Fig. 9 (b) shows the stage path that crossed the scanner area in and out. This case had lower stage acceleration. The processing speeds for the two cases were set high at $450 \mathrm{~mm} / \mathrm{s}$ and $500 \mathrm{~mm} / \mathrm{s}$.

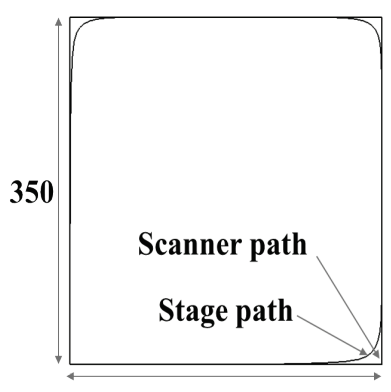

350

(a)

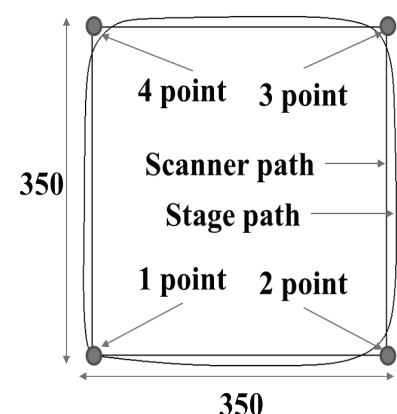

(b)
Fig. 9 Various stage paths using MICP. (a) when the stage path was at the center of the scanner and (b) when the stage path was in and out of the scanner center.

\subsection{Experiment Results}

To verify that continuous large-area processing was possible using the MICP path algorithm, signals were measured from the edge of the square. Figure 10 shows the measured data for the two-point section in Fig. 9 (a). Figure 10 (a) shows the results represented by the $\mathrm{X}$ axis, and Fig. 10 (b) shows those represented by the Y axis. The scanner made up for the area that the stage did not cover. The scanner did not go beyond the $50 \times 50 \mathrm{~mm}$ scan area; thus, it was verified that a large $350 \times 350 \mathrm{~mm}$ square was processed within the laser scanner area.

Figure 11 also shows the speed measured for each axis in the 2-point section. As seen in Fig. 11 (a), the stage speed on the $\mathrm{X}$ axis decreased near the edge. Then the scanner has been compensated in the opposite direction, and in Fig. 11 (b), the stage speed on the $\mathrm{Y}$ axis decreased near the edge. Then the scanner also has been compensated in the opposite direction.

The scanner moves so as to maintain change of the working speed of $400 \mathrm{~mm} / \mathrm{s}$ in accordance with the movement of the stage. Continuous large-area laser processing requires a constant processing speed. If the processing speed is not constant, the change processing line width. Therefore, there is no possible to obtain a good quality of product. In Fig. 11, the total processing speed was constant at $400 \mathrm{~mm} / \mathrm{s}$.

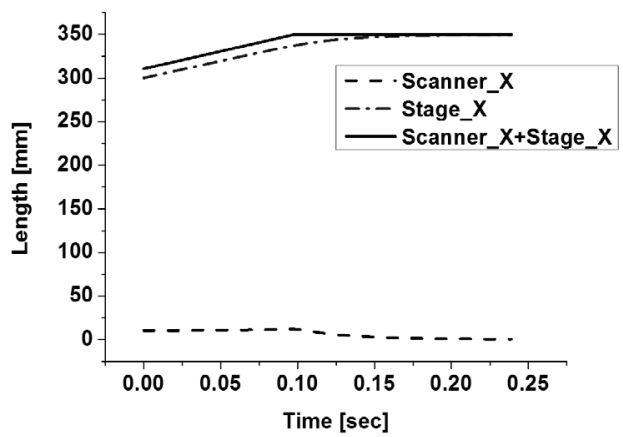

(a)

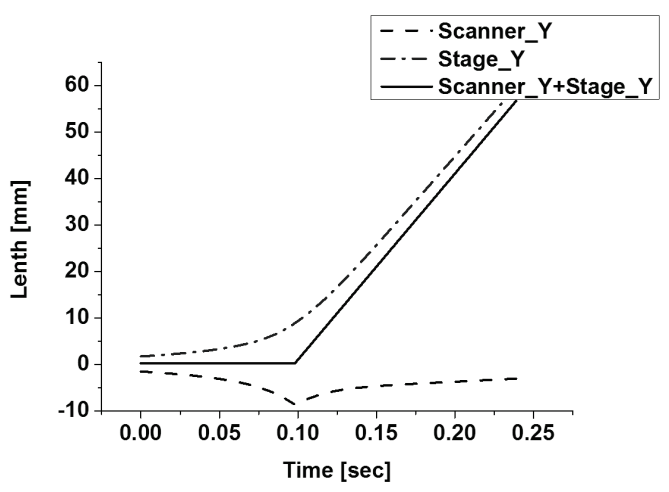

(b)

Fig. 10 Position results from the edge region: (a) X-axis and (b) Y-axis.

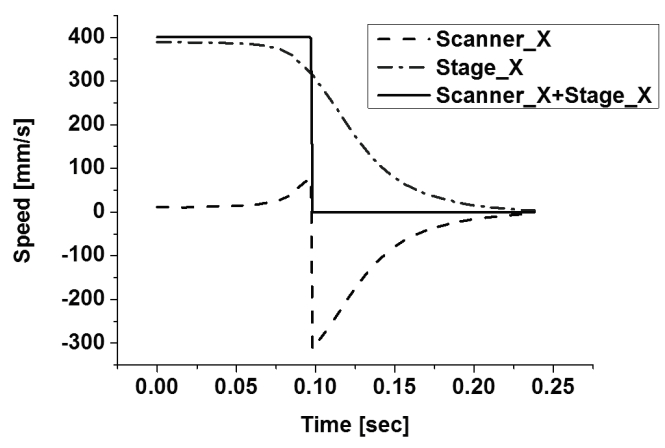

(a)

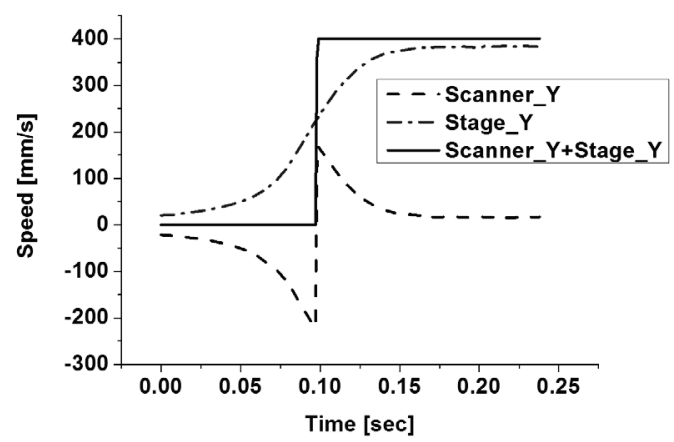

(b)

Fig. 11 Speed results from the edge region: (a) $\mathrm{X}$-axis and (b) $\mathrm{Y}$-axis. 


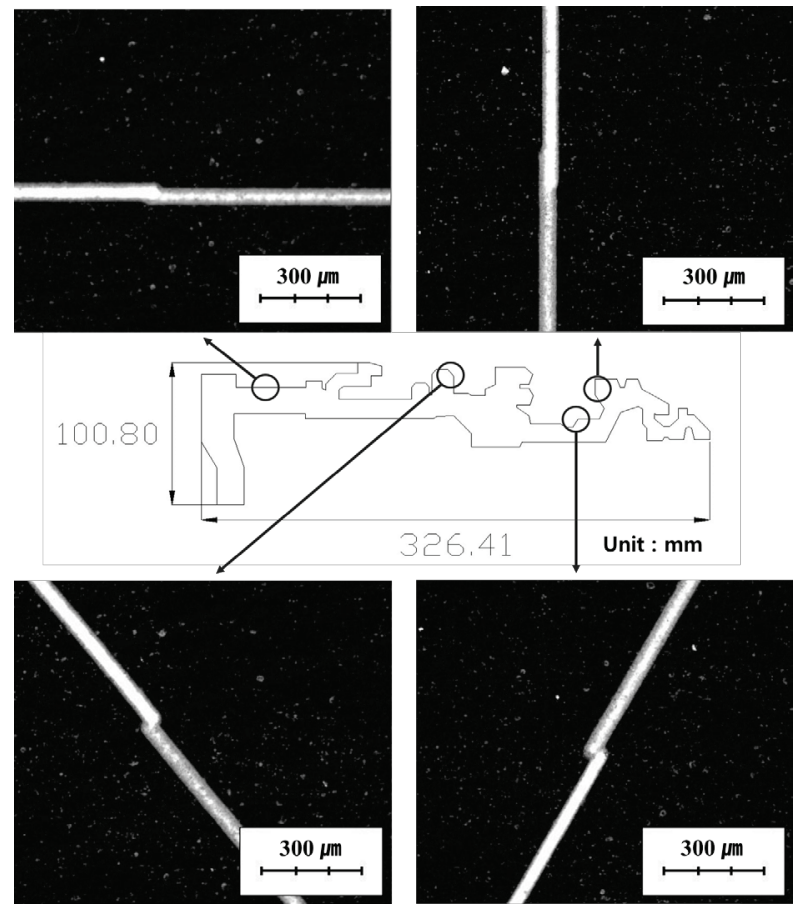

Fig. 12 Step \& scanning method for large-area processing.
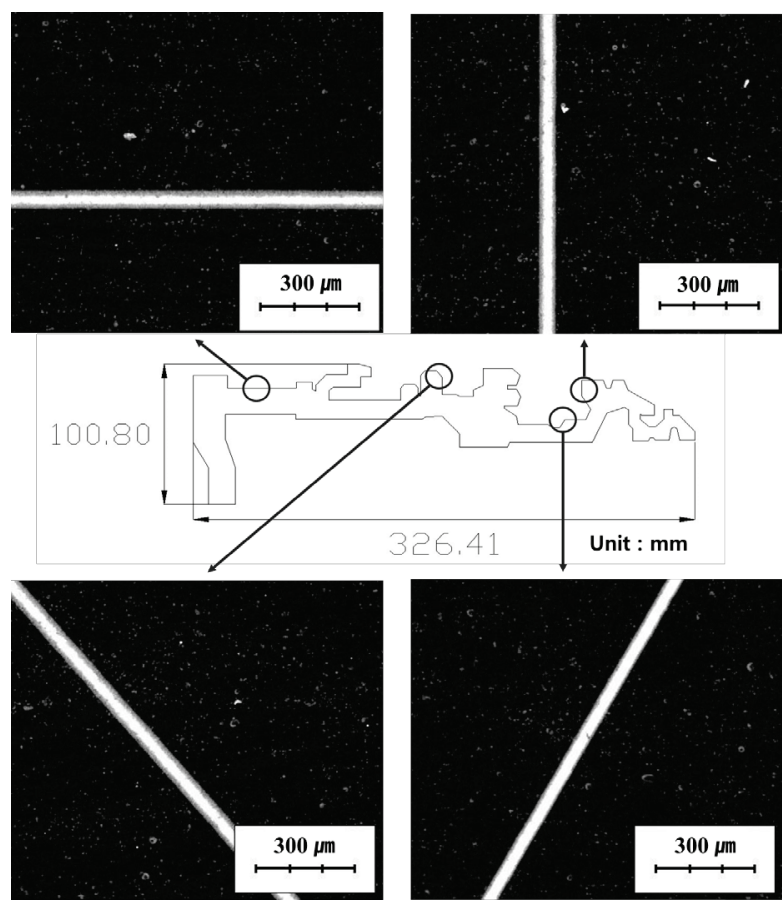

Fig. 13 MICP method for large-area processing.

Figures 12 and 13 illustrate the laser processing of a flexible printed circuit board (FPCB) pattern on an anodized aluminum plate specimen. Figure 12 shows the result of processing using conventional methods over a large area. In this step and scanning method, after scanning, the stage is moved. Accuracy is maintained in the scan area; however, the result is less accurate in the edge region. Figure 13 shows a processing result produced by the method proposed in this paper. It can be confirmed that the result is uniform in the edge region.
Table 1 The error at each point

\begin{tabular}{|c|c|c|c|c|}
\hline $\begin{array}{c}\text { Speed } \\
(\mathrm{mm} / \mathrm{s})\end{array}$ & Error & $(\mu \mathrm{m})$ & $\begin{array}{l}\text { Figure } 8 \\
\text { (a) Path }\end{array}$ & $\begin{array}{l}\text { Figure } 8 \\
\text { (b) Path }\end{array}$ \\
\hline \multirow{8}{*}{450} & \multirow{2}{*}{1 point } & $\mathrm{X}$ axis & 0 & 0 \\
\hline & & $\mathrm{Y}$ axis & 0 & 0 \\
\hline & \multirow{2}{*}{2 point } & $\mathrm{X}$ axis & 5.1 & 8.2 \\
\hline & & $\mathrm{Y}$ axis & 2.1 & 1.1 \\
\hline & \multirow{2}{*}{3 point } & $\mathrm{X}$ axis & -12.8 & -7.5 \\
\hline & & $\mathrm{Y}$ axis & 2.9 & -1.8 \\
\hline & \multirow{2}{*}{4 point } & $\mathrm{X}$ axis & 1.8 & 1.0 \\
\hline & & $\mathrm{Y}$ axis & -17.2 & -12.2 \\
\hline \multirow{8}{*}{500} & \multirow{2}{*}{1 point } & $\mathrm{X}$ axis & 0 & 0 \\
\hline & & $Y$ axis & 0 & 0 \\
\hline & \multirow{2}{*}{2 point } & $\mathrm{X}$ axis & 10.4 & 15.6 \\
\hline & & $Y$ axis & 2.0 & 1.0 \\
\hline & \multirow{2}{*}{3 point } & $\mathrm{X}$ axis & -20.9 & -5.0 \\
\hline & & $Y$ axis & 3.0 & -2.0 \\
\hline & \multirow{2}{*}{4 point } & $\mathrm{X}$ axis & 2.0 & 1.0 \\
\hline & & $Y$ axis & -33.9 & -28.7 \\
\hline
\end{tabular}

Table 1 shows measurements of the test specimens that were processed using the two stage paths shown in Fig. 9.

The stage path in Fig. 9 (b) had lower stage acceleration than that in Fig. 9 (a). The laser power was $2 \mathrm{~W}$ at $40 \mathrm{kHz}$, and the processing speed was $450 \mathrm{~mm} / \mathrm{s}$. The processed specimens were anodized aluminum plates, and the error was measured at each point. A higher speed led to greater errors. When the processing speed was $450 \mathrm{~mm} / \mathrm{s}$, the error was as much as $-17.2 \mu \mathrm{m}$ in the path in Fig. 9 (a), and -12.2 $\mu \mathrm{m}$ in the path in Fig. 9 (b). When the processing speed was $500 \mathrm{~mm} / \mathrm{s}$, the error was as much as $-33.9 \mu \mathrm{m}$ in the path in Fig. 9 (a), and $-28.7 \mu \mathrm{m}$ in the path in Fig. 9 (b). Near the edge, the error was smaller in the path in Fig. 9 (b), which had high stage acceleration, than in the path in Fig. 9 (b), which had low stage acceleration.

Table 2 shows the maximum errors of the horizontal and vertical measurements. The errors were greater in Fig. 9 (b) than in Fig. 9 (a); this indicates that the error was smaller when the stage path was the same as the scanner path. As seen in Tables 1 and 2, when the path was created using the MICP path algorithm, the error was smaller when the stage path coincided with the CAD path and when the path had lower acceleration/deceleration.

Table 2 Maximum vertical and horizontal error.

\begin{tabular}{|c|c|c|c|}
\hline $\begin{array}{c}\text { Speed } \\
(\mathbf{m m} / \mathbf{s})\end{array}$ & $\begin{array}{c}\text { Maximum } \\
\text { Error }(\boldsymbol{\mu m})\end{array}$ & $\begin{array}{c}\text { Figure 8 (a) } \\
\text { Path }\end{array}$ & $\begin{array}{c}\text { Figure 8 (b) } \\
\text { Path }\end{array}$ \\
\hline \multirow{2}{*}{450} & Horizontal & 2.3 & 18.2 \\
\cline { 2 - 4 } & Vertical & 1.2 & 10.4 \\
\hline \multirow{2}{*}{500} & Horizontal & 3.1 & 20.8 \\
\cline { 2 - 4 } & Vertical & 1.4 & 11.5 \\
\hline
\end{tabular}




\section{Conclusion}

In this study, the MICP path algorithm was developed for large-area laser patterning. The NURBS curve was used to easily generate the path using the mouse pointer. The stage speed was automatically calculated under constraint conditions, and a smooth speed profile was generated through speed distribution. There was no need for complicated G-code inputs, and the engineer's ideal paths were generated. The MICP path algorithm was used to generate a large-area square $(350 \times 350 \mathrm{~mm})$ that was larger than the $50 \times 50 \mathrm{~mm}$ scan area. Measurements verified that largearea processing was possible with a $50 \times 50 \mathrm{~mm}$ scan area at a constant processing speed of $400 \mathrm{~mm} / \mathrm{s}$. In addition, a processing experiment was conducted with the paths wherein the scan path and the stage path were the same and with paths with lower acceleration/deceleration. When the processing speed was $450 \mathrm{~mm} / \mathrm{s}$ or $500 \mathrm{~mm} / \mathrm{s}$, the error was smaller when the stage path was the same as the scanner path, and when the acceleration/deceleration was low. It is expected that this algorithm will be used for easy laser processing of various patterns.

\section{References}

[1] Kim, T., Park, S., Oh, H. and Shin, Y., "Analysis of the laser patterning inside light guide panel," Optics \& Laser Technology, Vol. 39, No. 7, pp. 1437-1442, 2007

[2] Nikumb, S., Chen, Q., Li, C., Reshef, H., Zheng, H. Y., Qiu H. and Low, D., "Precision glass machining, drilling and profile cutting by short pulse lasers," Thin Solid Films, Vol. 477, No. 1-2, pp. 216-221, 2007.

[3] Shin, D., Lee J., Sohn, H., Noh J., and Paik, B., A FPCB cutting process using a pico-second laser, JLMN, Vol. 5,. No. 1, pp. 48-52, 2010
[4] Erkorkmaz, K., Alzaydi, A., Elfizy, A., Engin, S., "Time-optimal trajectory generation for 5-axis on-thefly laser drilling", CIRP Annals - Manufacturing Technology, Vol.60, Issue 1, pp.411-414, 2011

[5] Hatwig J., Reinhart G, Zaeh MF., "Automated task planning for industrial robots and laser scanners for remote laser beam welding and cutting", Pod. Eng. Vol. 4, Issue 4, pp 327-332, 2010

[6] Kim, K. H., Lee, J. H. and Suh, J., "Laser scanner stage on the fly technique for high precision/ultrafast/wide area fabrication," Proc. of KSME Spring Conf., pp. 31-32, 2010.

[7] Yoon, K. H., Lee, J. H., Kim K. H. and Suh, J., "Scanner-Stage Synchronization Control Method for Laser Fabrication of Large Aera," Proc. of KSME Spring Conf., pp. 287-288, 2010.

[8] Kim, K. H., Lee, J. H., Suh, J. and Yoon, K. H., "The scanner-stage on the fly technology for a ultraprecision/ultrafast/wide area fabrication," Proc. of KSLP Spring Conf., pp. 44-46, 2010.

[9] Lee, J. H. and Kim, K. H., "Core technology for ultrafast/ wide area laser processing," Machinery and Materials, Vol. 22, No. 1, pp. 36-42, 2010.

[10] Kim, K. H., Lee, J. H., and Yoon, K. H., "Path Generation Algorithm Development for Ultrafast/Wide Area Laser Processing," Journal of the KSPE, Vol. 27, No. 10, pp.34-39, 2010

[11] Nam, J. H., "Construction of NURBS Model for Preliminary High-Speed Monohull Design Based on Parametric Approach," J. of Ocean Engineering and Technology, Vol. 20, No. 3, pp. 82-87, 2006.

[12] ESI, http://www.esi.com/

[13] LPKF, http://www.lpkf.com/

[14] AEROTECH, http://www.aerotech.com/

(Received: March 31, 2014, Accepted: March 26, 2015) 